\title{
Construcción y validación del Cuestionario de Violencia de Pareja en jóvenes de Lima Este
}

\section{Construction and validation of the Couple Violence Questionnaire in young people from East Lima}

\author{
Dayan Arroyo Chávez, Maryori Riquez Julca, Cristian Adriano Rengifo
}

\begin{abstract}
RESUMEN
Objetivo: construir y evaluar de las propiedades psicométricas de un instrumento que evalúa la dinámica de pareja dentro de una relación de jóvenes entre las edades de 18 y 30 años. Metodología: Después de realizar una estricta revisión teórica y haber definido el constructo, se elaboró el Cuestionario de Violencia de Parejas en Jóvenes, con 29 ítems, con sus 5 factores: factor 1 Violencia Física (10 ítems), factor 2 Manipulación (5 ítems), factor 3 Violencia Verbal (6 ítems), factor 4 Coerción (3 ítems); por último, el factor 5 Prohibiciones ( 5 ítems); con un tipo de respuesta dicotómica y Likert de cinco puntos. Fue administrado a 355 jóvenes residentes de Lima- Este. Para evaluar la validez de constructo se hizo mediante el Análisis Factorial Exploratorio, utilizando el método de componentes paralelos con rotación oblimin y la normalización Kaiser-Meyer-Olkin (KMO). Resultados: se obtuvo un KMO de .937, con una significancia de .001; la rotación indicó que cinco factores se ajustan adecuadamente, con pesos factoriales dentro del rango de .350 a .623 , que representa el $53.5 \%$ de la varianza. Asimismo, se obtuvo un alfa de Cronbach de .943 y un omega de McDonald's .947 en la escala global; así también, en los ítems se evidencia coeficientes de Cronbach que varían entre .940 a .942 y en el McDonald's .944 a .946, lo que indica una elevada fiabilidad. Conclusión: el instrumento evidencia una buena consistencia interna y validez de constructo.
\end{abstract}

Palabras clave: Dinámica, violencia, pareja, jóvenes.

\section{ABSTRACT}

Objective: to construct and evaluate the psychometric properties of an instrument that assesses couple dynamics within a relationship of young people between the ages of 18 and 30 years. Methodology: After carrying out a strict theoretical review and having defined the construct, the Youth Violence Questionnaire with 29 items was prepared, with its 5 factors: factor 1 Physical Violence (10 items), factor 2 Manipulation (5 items ), factor 3 Verbal Violence (6 items), factor 4 Coercion (3 items); finally, the factor 5 Prohibitions ( 5 items); with a type of dichotomous response and Likert of five points. It was administered to 355 young residents of Lima-Este. The construct validity was evaluated by means of the Exploratory Factor Analysis, using the method of parallel components with oblimin rotation and the Kaiser-Meyer-Olkin (KMO) normalization. Results: a KMO of .937 was obtained, with a significance of .001; The rotation indicated that five factors are adequately adjusted, with factor weights within the range of .350 to .623 , which represents $53.5 \%$ of the variance. Likewise, a Cronbach's alpha of .943 and a McDonald's omega .947 were obtained on the global scale; likewise, the items show Cronbach coefficients that vary between .940 to .942 and McDonald's .944 to .946 , indicating high reliability. Conclusion: the instrument shows good internal consistency and construct validity.

Keywords: Psychometrics, dynamics, couples, young people.

${ }^{1}$ Universidad Peruana Unión, Lima, Perú

Orcid ID: 0000-0001-9211-511X 


\section{INTRODUCCIÓN}

La violencia en el Perú es uno de los problemas sociales que ha ido aumentando a lo largo de los años y ocasionan severas consecuencias, no sólo a la víctima, sino también a la familia de esta. El Instituto Nacional de Estadística e Informática (INEI,2019) registró 14,491 casos de violencia contra la mujer, violencia familiar y violencia sexual en el mes de enero del presente año, por medio de los Centros de Emergencia Mujer (CEM) a nivel nacional. Así mismo se realiza una comparación con el reporte desde enero hasta el mes de julio, y se reportó 101,668 casos a nivel nacional, lo que demuestra que es un problema psicosocial que va aumentando de manera rápida.

Pazos, Oliva y Hernando (2014) definen la violencia en la relación de pareja como una agresión intencional por parte de un miembro de la relación, quien posee mayor autoridad y poder hacia la pareja, ya sea de manera física, psicológica o sexual. En los adolescentes y jóvenes es más probable que se presenten conductas de violencia, ya que tienen dificultad para poder identificar que son víctimas de la violencia por parte de su pareja, e idealizan la relación a tal punto de normalizar las conductas agresivas.

También, Muñoz y Echeburúa (2016) clasifican la violencia de pareja en tres tipos: violencia física, psicológica y sexual, y hacen referencia que la violencia física y sexual van de la mano del tipo psicológico, debido a que este último es como empieza la violencia, tiene consecuencias más graves y es más difícil de detectar debido a que son comportamientos que pasan desapercibidos, se muestra de diferentes maneras y hasta se puede confundir con expresiones de "amor", por ejemplo: control de redes sociales, celos, control de la otra persona, violencia verbal, aislamiento, confusión emocional, sometimiento económico, acoso, etc.

Rodríguez y Biezma (2017) menciona varios tipos de violencia en la pareja: la violencia física, violencia psicológica, violencia sexual. El primer tipo son conductas agresivas como jaloneos, golpes, empujones que generan lesiones en el organismo, hasta puede llegar a la muerte. En el segundo tipo, se caracteriza por los insultos, amenaza o calificativos negativos y discriminatorios, humillación. El tipo sexual se centra generalmente en la obligación del contacto sexual sin el consentimiento de la otra persona, lo cual muchas veces va acompañado de agresiones físicas.

La violencia en las relaciones de parejas jóvenes empieza de manera gradual y progresiva, con conductas, mayormente de tipo psicológico, con el fin de que pase desapercibido y tener el control sobre la pareja. Los factores que ayudan a que la violencia se mantenga son los mitos 0 creencias que las personas adoptan, como por ejemplo de que la agresión física es la solución a los problemas, los estereotipos ya establecidos por la sociedad; también influye si en sus familias respectivas han observado esas conductas violentas y han crecido con ese modelo, de manera que existen dos posibilidades: que repitan esas conductas de violencia o sumisión; y la otra cambiar esas conductas violentas. Otro factor importante con respecto al mantenimiento de la violencia son las características de la relación, ya que, según varios estudios realizados, mientras mayor sea el tiempo de relación, el contacto e intimidad sexual y que tan consolidada esté la relación, mayor es el riesgo de la presencia de la violencia, en especial del tipo psicológico (Rubio-Garay et al., 2015).

Muchas veces, la violencia que ejerce el agresor está relacionada con factores individuales y características de la personalidad de este, como: impulsividad, desconfianza, poca tolerancia a la frustración, poca capacidad para resolver problemas, entre otras; y conductas de riesgo, como el consumo del alcohol, drogas, inicio sexual a corta edad, problemas en la familia, bajo rendimiento escolar, etc. (Pazos, Oliva y Hernando, 2014).

García , et al (2018) tomando los resultados de la Macroencuesta de violencia contra la mujer realizada en España el 2015, reportan que la violencia psicológica tiene mayor prevalencia $(38.3 \%)$ en las jóvenes entre las edades 16 a 24 años, en comparación a las mujeres jóvenes mayores de 24 años (25.43\%). Así también, diferentes estudios explican que las mujeres entre las edades de 20 a 24 años y que se encuentran en la etapa universitaria son más propensas a sufrir cualquier tipo de violencia por parte de sus parejas.

En España, García, Rodríguez y Porcel (2018) validaron la Escala Multidimensional de Violencia en el Noviazgo (EMVN), finalidad de detectar la violencia a manera de víctima y agresor en la 
relación de pareja. Este consta de 32 ítems, con tres dimensiones: agresiones físicas y sexuales; conductas de control y abuso psicoemocional. La muestra estuvo compuesta de 447 estudiantes de ambos sexos. El estudio demuestra que las mujeres recuerdan acontecimientos del pasado a manera agresiva, están más pendientes de sus parejas en las redes sociales, pero por otro lado reciben mensajes controladores por parte de los hombres, asimismo ellos se enfadan con más frecuencia e intensidad.

Otro de los instrumentos, fue realizado con estudiantes provenientes de diferentes centros de estudios de España, México y Argentina con la Cuestionario de Violencia entre Novios (CUVINO) creado por Rodríguez et al, (2010) finalidad de evaluar y detectar hechos de violencia en parejas jóvenes universitarios. Este consta de 42 ítems, con un formato Likert de cinco categorías de respuestas $(0=$ Nunca, 1 = a veces, 2 = frecuente, 3 = habitual y 4 = casi siempre) y se compone de ocho dimensiones: el castigo emocional, la coerción, el desapego, el maltrato físico, género, la humillación, la violencia instrumental y la violencia sexual. La muestra estuvo compuesta de 5170 participantes de ambos sexos que oscilan entre las edades de 15 y 26 años (Sánchez, 2017). Este cuestionario ha sido utilizado en una población peruana, la muestra estuvo compuesta por un total de 595 estudiantes de ambos sexos considerando que han tenido una relación de noviazgo en los últimos doce meses. Los resultados muestran que el cuestionario es confiable siendo que es mayor a .959 en Alfa de Cronbach, además la confiabilidad en las dimensiones varía entre .641 y .860. Asimismo, la aportación de este cuestionario CUVINO es evaluar la violencia entre pareja, para así prevenir y hacer frente a la situación (León, 2017).

Se han propuesto varios instrumentos para medir la violencia en el noviazgo, tales como el Inventario de Violencia en las Relaciones de Noviazgo entre Adolescente (CADRI) creada por Wolfe, consta de seis dimensiones: violencia física, amenazas, violencia verbal emotiva, violencia sexual, violencia relacional. Este cuestionario ha sido utilizado en una población peruana, la muestra estuvo compuesta por un total de 515 estudiantes de ambos sexos (Villena, 2016).

La escala de Violencia en la Relación de Pareja en Jóvenes (E.V.R.P) creada por Bejarano y
Vega (2014) en Perú, presenta tres dimensiones: violencia física, violencia psicológica y violencia sexual.

Finalmente, el objetivo principal de esta investigación es construir y evaluar las propiedades psicométricas del Cuestionario de Violencia de Pareja en jóvenes, de modo que se pueda identificar las conductas violentas en las relaciones afectivas de los jóvenes, ya sea de tipo física, psicológica y/o sexual, de manera que sean conscientes y reconocer dichas conductas como tal.

\section{METODOLOGÍA}

El estudio realizado es de enfoque cuantitativo, con diseño no experimental y de corte transversal, debido a que la variable no se ha manipulado y se recolecta la información una sola vez (Tam, Vera y Oliveros, 2008). Además, esta investigación es de diseño instrumental, ya que se construye un instrumento de medición psicológica y se analizan las propiedades psicométricas de dicho instrumento (Ato, López y Benavente, 2013).

\section{Participantes}

Para esta investigación se contó con una muestra de 355 jóvenes, que fueron seleccionados a través de un muestreo no probabilístico por conveniencia, ya que los jóvenes que se encuentran actualmente en una relación de pareja responden activamente a la invitación de participar en el estudio (Hernández et al., 2010). En la muestra fueron incluidos 355 jóvenes de ambos sexos residentes de Lima Este, se evaluaron 213 jóvenes del sexo femenino $(60.0 \%)$ y del sexo masculino fueron 142 jóvenes (40.0\%), con edades entre 18 a 30 años con una edad promedio de 22.1 años (DS = 3.18). Seleccionados porque se encontraban en una relación de pareja, divididos de la siguiente manera: 152 jóvenes que tienen una relación de menos de 1 año (42.8\%), 92 jóvenes que se encuentran en una relación de 1 a 2 años (25.9\%), 59 personas que tienen una relación de 2 a 3 años (16.6\%) y 52 jóvenes con más de 3 años de relación (14.6\%). Además, se tuvo en cuenta el lugar de procedencia, identificándose 245 jóvenes de la costa (69.0\%), 73 jóvenes de la sierra $(20.6 \%), 33$ de la sierra $(9.3 \%)$ y 4 jóvenes del extranjero (1.1\%). 


\section{Procesamiento de datos}

Para la construcción del instrumento se realizó una revisión teórica exhaustiva sobre el constructo a estudiar: violencia de pareja en jóvenes, lo que es un paso fundamental para construir el instrumento psicométrico (Hernández et al., 2010). Después se procedió a la elaboración de 42 ítems, proponiéndole 3 dimensiones: Violencia Física, Violencia Psicológica y Violencia Sexual. El instrumento es valorado en una escala de tipo Likert, (1 al 5) $1=\operatorname{Nunca}(\mathrm{N}), 2=$ Casi Nunca $(\mathrm{CN}), 3=$ A veces $(\mathrm{AV}), 4=$ Casi siempre (CS) y $5=$ Siempre (S).

Al término de la construcción de ítems, con el fin de avalar el instrumento, contenido y estructura se sometió a la revisión de una comisión de expertos, conformados por cuatro jueces: por dos psicólogos clínicos especialistas en diagnósticos y psicoterapia, asimismo una psicóloga clínica con especialidad en violencia, por último, se contó con una psicóloga especialista en investigación. Los ítems de la escala se evaluaron sobre la base de cuatro criterios, claridad, congruencia, contexto y dominio de constructo. Para la cuantificación se usó $\mathrm{V}$ de Aiken.

El instrumento psicométrico "Cuestionario de Violencia de Parejas en jóvenes", quedó constituido por 42 ítems, dividido en tres dimensiones: violencia psicológica (26 ítems); violencia física (11 ítems) y violencia sexual (5 ítems). Al momento de aplicar el cuestionario, un gran porcentaje no respondió los ítems de la dimensión sexual $(26,27,28,29$ y 30 ), ya que para responder dichos ítems los evaluados debían haber tenido contacto sexual previamente. Además, al realizar el análisis factorial exploratorio, se agrupó en 5 factores: violencia física, manipulación, violencia verbal, coerción y prohibición. Sin embargo, se eliminaron 5 ítems: 5, 11, 12, 31 y 32 por revisión teórica, quedando así con 32 ítems.

Para la validez del constructo se realizó el análisis factorial exploratorio de la escala, para ello se utilizó el método de componentes paralelos con rotación oblimin. Además, antes de este proceso se presenta el cumplimiento de los criterios de la prueba de esfericidad de Bartlett y el índice KMO (Káiser-Meyer-Oikin). Finalmente se llevó a cabo el análisis de fiabilidad en la muestra piloto, evaluándose por el método de la consistencia interna, para el que se empleó Alpha coeficiente de Cronbach y la Omega de McDonald.

\section{RESULTADOS}

\section{Validez de contenido}

Para usar los ítems, es esencial realizar un proceso de revisión teórica para los componentes del constructo, Soto \& Segovia (2009) mencionan que los reactivos cuyos valores sean mayores o iguales a 0.75 se consideran válidos para el test, de esta manera se procedió a calcular los coeficientes mediante el método de validez $\mathrm{V}$ de Aiken para el test, donde los ítems presentan valores mayores de 0.75 (ver tabla 1), lo cual indica la validez del contenido del instrumento, ya que existe relación con los ítems, las palabras están acorde al contexto y evalúa lo que pretende.

En la tabla 1, se puede observar que la validez del contenido por criterios de jueces mediante la $\checkmark$ de Aiken tiene validez de 0.75 , lo que significa que hay claridad, congruencia, contexto y dominio en la redacción de los ítems. 
Tabla 1

Análisis de la validez de contenido del instrumento.

\begin{tabular}{|l|l|}
\hline Test & V-Aiken \\
\hline Forma correcta de aplicación y estructura & .75 \\
\hline Orden de las preguntas establecido adecuadamente & .75 \\
\hline Contiene el test preguntas difíciles de entender & .75 \\
\hline Contiene el test palabras difíciles de entender & 1 \\
\hline Las opciones de respuestas son pertinentes y están suficientemente graduados & .75 \\
\hline Los ítems del instrumentos corresponden a la dimensión del constructo & 1 \\
\hline
\end{tabular}

\section{Validez de constructo}

Con el objetivo de verificar la validez del constructo de violencia en parejas, se consideró necesario usar el análisis factorial exploratorio, ya que es la técnica más usada para el desarrollo de un cuestionario, debido a que se verifica la cantidad de factores que explican las respuestas de cada ítem del cuestionario en general (Lloret, Ferreres, Hernández y Tomás, 2014). Antes considerar los componentes que resultaron del análisis factorial se corroboró la idoneidad de muestreo (KMO) fue de .937 [ver tabla 2], donde indican que los datos tienen una muestra apropiada siendo que es $>.80$ donde existe una alta correlación de las variables y la muestra es adecuada para poder aplicar el análisis factorial exploratorio (Escurra y Salas, 2014). Asimismo, la prueba de esfericidad de Bartlett contrasta la hipótesis de que la matriz de correlaciones es igual, es decir, los ítems no se repiten por lo que se puede realizar el análisis factorial exploratorio (AFE) (Escurra et al., 2014).
Solo se usaron 29 de los 32 ítems del instrumento, debido a que el ítem 33 “¿Pide permiso a su pareja antes de salir a algún lugar?" no cargó en ningún factor, los ítems inversos (8 y 40) también se eliminaron por efecto de método, por otro lado los ítems 15 , 17,19 y 24 presentaban cargas factoriales en Violencia física y Prohibición pero por definición conceptual se ubicaron en el primer factor; el ítem 4 presentó carga en el segundo y tercer factor, quedándose en el tercer factor: Violencia Verbal y los ítems 10 y 42 presentaron cargas factoriales en los factores 3, 4 y 5, pero debido a la definición conceptual se decidió mantenerse en el factor 5: Prohibición

Se realizó el análisis factorial sobre los ítems, usando componentes paralelos y rotación oblimin. Los resultados de estos análisis indicaron que se ajusta en cinco factores adecuadamente, evidenciándose a cada ítem con una fuerte carga factorial. La tabla 3 muestra que los 29 ítems se agrupan en 5 factores, en el factor Violencia Física se agrupan los ítems: $15,17,18,19,20,21,22,23,24$ y 25 , tienen una carga dentro del rango de .311 - .736; en el factor 2 Manipulación se encuentran: 7, 36, 37, 38 y 39 , con carga en el rango de .405 - .769; el factor 3 Violencia Verbal está conformado por los ítems: 1, 2, 3, 4, 6 y 9, cuya carga oscila entre .408 - .829; en el factor 4 Coerción se encuentran: 34,35 y 41, con carga entre 605 y .746; por último, el factor 5 Prohibiciones cuenta con los ítems: 10, 13, 14, 16 y 42, con una carga dentro del rango .350 - .623, representando el $53.5 \%$ de la varianza acumulada. 
Tabla 3

Matriz de factores rotados del constructo

\begin{tabular}{|c|c|c|c|c|c|c|}
\hline & \multicolumn{6}{|c|}{ Factores } \\
\hline & 1 & 2 & 3 & 4 & 5 & Uniqueness \\
\hline VP1_r & & & 0.517 & & & 0.588 \\
\hline VP2_r & & & 0.629 & & & 0.403 \\
\hline VP3_r & & & 0.829 & & & 0.265 \\
\hline VP4_r & & 0.348 & 0.408 & & & 0.633 \\
\hline VP6_r & & & 0.423 & & & 0.544 \\
\hline VP7_r & & 0.529 & & & & 0.586 \\
\hline VP9_r & & & 0.486 & & & 0.505 \\
\hline VP10_r & & & 0.308 & & 0.530 & 0.436 \\
\hline VP13_r & & & & & 0.385 & 0.627 \\
\hline VP14_r & & & & & 0.432 & 0.613 \\
\hline VP15_r & 0.311 & & & & 0.372 & 0.520 \\
\hline VP16_r & & & & & 0.623 & 0.403 \\
\hline VP17_r & 0.331 & & & & 0.380 & 0.424 \\
\hline VP18_r & 0.586 & & & & & 0.489 \\
\hline VP19_r & 0.427 & & & & 0.358 & 0.436 \\
\hline VP20_r & 0.487 & & & & & 0.408 \\
\hline VP21_r & 0.621 & & & & & 0.450 \\
\hline VP22_r & 0.736 & & & & & 0.336 \\
\hline VP23_r & 0.659 & & & & & 0.290 \\
\hline VP24_r & 0.387 & 0.509 & & & & 0.354 \\
\hline VP25_r & 0.414 & & & & & 0.702 \\
\hline VP34_r & & & & 0.678 & & 0.270 \\
\hline VP35_r & & & & 0.746 & & 0.264 \\
\hline VP36_r & & 0.405 & & & & 0.655 \\
\hline VP37_r & & 0.651 & & & & 0.413 \\
\hline VP38_r & & 0.769 & & & & 0.302 \\
\hline VP39_r & & 0.673 & & & & 0.550 \\
\hline VP41_r & & & & 0.605 & & 0.466 \\
\hline VP42_r & & & & 0.452 & 0.350 & 0.551 \\
\hline
\end{tabular}

Note. 'Minimum residual' extraction method was used in combination with a 'oblimin' rotation

\section{Fiabilidad}

Para confirmar la fiabilidad del cuestionario se valoró calculando el índice de la consistencia interna mediante el coeficiente Alpha de Cronbach y Omega de McDonald's, en la tabla 4 se puede observar que la consistencia interna del Cuestionario de Violencia de Pareja en jóvenes (29 ítems) en la muestra estudiada alcanza un coeficiente de .943 en Cronbach y .947 de McDonald's, lo que indica elevada fiabilidad. Del mismo modo, en la tabla 5 se observa que en relación a los ítems se evidencia coeficientes de Cronbach que varían entre .940 a .942 los cuales afirman elevada fiabilidad siendo que es mayor a .90 (Hernández, Fernández, y Baptista, 2010) y en el McDonald's .944 a .946 el valor adecuado de la confiabilidad es de .70 y .90 (Campo y Oviedo, 2008). 
Tabla 4

Análisis de fiabilidad del constructo

\begin{tabular}{lllll} 
& Media & D.E. & Cronbach's a & McDonald's w \\
Escala global & 1.55 & 0.571 & 0.943 & 0.947 \\
\hline
\end{tabular}

Tabla 5

Análisis de fiabilidad por ítems

\begin{tabular}{|c|c|c|}
\hline & Cronbach's a & McDonald's $\omega$ \\
\hline VP1_r & 0.942 & 0.946 \\
\hline VP2 ${ }^{-} r$ & 0.940 & 0.945 \\
\hline VP3 $r$ & 0.940 & 0.945 \\
\hline VP4_r & 0.942 & 0.946 \\
\hline VP6_r & 0.941 & 0.945 \\
\hline VP7_r & 0.942 & 0.946 \\
\hline VP9_r & 0.941 & 0.945 \\
\hline VP10_r & 0.941 & 0.945 \\
\hline VP13r & 0.941 & 0.946 \\
\hline VP14_r & 0.942 & 0.946 \\
\hline VP15_r & 0.941 & 0.945 \\
\hline VP16_r & 0.940 & 0.945 \\
\hline VP17_r & 0.940 & 0.944 \\
\hline VP18_r & 0.941 & 0.945 \\
\hline VP19_r & 0.941 & 0.945 \\
\hline VP20_r & 0.941 & 0.945 \\
\hline VP21_r & 0.941 & 0.945 \\
\hline VP22_r & 0.940 & 0.944 \\
\hline VP23_r & 0.940 & 0.944 \\
\hline VP24_r & 0.941 & 0.944 \\
\hline VP25_r & 0.942 & 0.946 \\
\hline VP33_r & 0.942 & 0.946 \\
\hline VP34_r & 0.940 & 0.944 \\
\hline VP35_r & 0.940 & 0.945 \\
\hline VP36_r & 0.942 & 0.946 \\
\hline VP37_r & 0.941 & 0.945 \\
\hline VP38_r & 0.941 & 0.945 \\
\hline VP39_r & 0.942 & 0.946 \\
\hline VP41_r & 0.942 & 0.946 \\
\hline VP42_r & 0.942 & 0.946 \\
\hline
\end{tabular}

Tabla

Ítems de cuestionario

\begin{tabular}{|c|c|c|c|c|c|c|c|}
\hline Antes & Después & Preguntas & \multicolumn{5}{|c|}{ Respuestas } \\
\hline 1 & 1 & $\begin{array}{l}\text { ¿Su pareja le prohíbe subir ciertas fotos a sus redes } \\
\text { sociales? }\end{array}$ & $\mathrm{N}$ & $\mathrm{CN}$ & AV & CS & $\mathrm{S}$ \\
\hline 2 & 2 & ¿Su pareja le grita? & $\mathrm{N}$ & $\mathrm{CN}$ & AV & CS & $\mathrm{S}$ \\
\hline 3 & 3 & ¿Su pareja le ha insultado? & $\mathrm{N}$ & $\mathrm{CN}$ & AV & CS & $\mathrm{S}$ \\
\hline 4 & 4 & ¿Su pareja le ha maldecido? & $\mathrm{N}$ & $\mathrm{CN}$ & AV & CS & $\mathrm{S}$ \\
\hline 6 & 5 & ¿Su pareja le dice apodos ofensivos y desagradables? & $\mathrm{N}$ & $\mathrm{CN}$ & AV & CS & $\mathrm{S}$ \\
\hline 7 & 6 & $\begin{array}{l}\text { ¿En alguna ocasión su pareja le prohibió la visita de sus } \\
\text { familiares? }\end{array}$ & $\mathrm{N}$ & $\mathrm{CN}$ & AV & CS & S \\
\hline 9 & 7 & ¿Su pareja le dice palabras que le hacen sentir mal? & $\mathrm{N}$ & $\mathrm{CN}$ & AV & CS & $\mathrm{S}$ \\
\hline 10 & 8 & ¿Su pareja le prohíbe hablar con ciertas personas? & $\mathrm{N}$ & $\mathrm{CN}$ & $\mathrm{AV}$ & CS & $\mathrm{S}$ \\
\hline 13 & 9 & $\begin{array}{l}\text { ¿Su pareja le prohíbe usar prendas de vestir de su } \\
\text { agrado? }\end{array}$ & $\mathrm{N}$ & $\mathrm{CN}$ & $\mathrm{AV}$ & CS & S \\
\hline 14 & 10 & $\begin{array}{l}\text { ¿Su pareja le hace problema cuando sale con sus } \\
\text { amistades? }\end{array}$ & $\mathrm{N}$ & $\mathrm{CN}$ & $\mathrm{AV}$ & CS & S \\
\hline 15 & 11 & $\begin{array}{l}\text { ¿Ha recibido pellizcos de su pareja cuando se molesta } \\
\text { con usted? }\end{array}$ & $\mathrm{N}$ & $\mathrm{CN}$ & AV & CS & S \\
\hline 16 & 12 & $\begin{array}{l}\text { ¿Su pareja le prohíbe realizar ciertas actividades que a } \\
\text { usted le gustan? }\end{array}$ & $\mathrm{N}$ & $\mathrm{CN}$ & $\mathrm{AV}$ & CS & $\mathrm{S}$ \\
\hline
\end{tabular}




\begin{tabular}{|c|c|c|c|c|c|c|c|}
\hline 17 & 13 & ¿Recibe jaloneos o manotazos por parte de su pareja? & $\mathrm{N}$ & $\mathrm{CN}$ & AV & CS & $S$ \\
\hline 18 & 14 & ¿Recibe alguna cachetada por parte de su pareja? & $\mathrm{N}$ & $\mathrm{CN}$ & AV & CS & S \\
\hline 19 & 15 & ¿Su pareja le jala el cabello? & $\mathrm{N}$ & $\mathrm{CN}$ & AV & CS & $S$ \\
\hline 20 & 16 & ¿Su pareja le araña? & $\mathrm{N}$ & $\mathrm{CN}$ & AV & CS & S \\
\hline 21 & 17 & ¿En situaciones su pareja le tiró algún objeto? & $\mathrm{N}$ & $\mathrm{CN}$ & AV & CS & S \\
\hline 22 & 18 & ¿En ocasiones su pareja la/o empujó? & $\mathrm{N}$ & $\mathrm{CN}$ & AV & CS & $S$ \\
\hline 23 & 19 & ¿En ocasiones hubo golpes por parte de su pareja? & $\mathrm{N}$ & $\mathrm{CN}$ & AV & CS & S \\
\hline 24 & 20 & ¿En discusiones con su pareja existen patadas? & $\mathrm{N}$ & $\mathrm{CN}$ & AV & CS & S \\
\hline 25 & 21 & ¿Recibe mordidas por parte de su pareja? & $\mathrm{N}$ & $\mathrm{CN}$ & AV & CS & $S$ \\
\hline 34 & 22 & $\begin{array}{l}\text { ¿Su pareja le amenaza con terminar la relación si usted } \\
\text { no hace lo que él/ella desea? }\end{array}$ & $\mathrm{N}$ & $\mathrm{CN}$ & AV & CS & $S$ \\
\hline 35 & 23 & ¿Su pareja le amenaza con dejarla/o? & $\mathrm{N}$ & $\mathrm{CN}$ & AV & CS & $S$ \\
\hline 36 & 24 & $\begin{array}{l}\text { ¿Su pareja amenazó con hacerse o hacerle daño si la/lo } \\
\text { dejaba? }\end{array}$ & $\mathrm{N}$ & $\mathrm{CN}$ & AV & CS & S \\
\hline 37 & 25 & ¿Su pareja le pide dinero sin razón alguna? & $\mathrm{N}$ & $\mathrm{CN}$ & AV & CS & $S$ \\
\hline 38 & 26 & $\begin{array}{l}\text { ¿Su pareja le dice que si no le compra lo que quiere se va } \\
\text { a buscar a otro(a)? }\end{array}$ & $\mathrm{N}$ & $\mathrm{CN}$ & AV & CS & $S$ \\
\hline 39 & 27 & $\begin{array}{l}\text { ¿Su pareja le exige que solvente actividades costosas } \\
\text { (viajes, ropa, comida, etc.)? }\end{array}$ & $\mathrm{N}$ & $\mathrm{CN}$ & AV & CS & S \\
\hline 41 & 28 & $\begin{array}{l}\text { ¿Su pareja le hace sentir que usted tiene la culpa cuando } \\
\text { discuten? }\end{array}$ & $\mathrm{N}$ & $\mathrm{CN}$ & AV & CS & $S$ \\
\hline 42 & 29 & $\begin{array}{l}\text { ¿su pareja le pide que deje sus actividades personales } \\
\text { para estar con él/ella? }\end{array}$ & $\mathrm{N}$ & $\mathrm{CN}$ & $\mathrm{AV}$ & CS & $S$ \\
\hline
\end{tabular}

\section{DISCUSIÓN}

La violencia de pareja en jóvenes se define como una agresión intencional por parte de un miembro de la relación, quien posee mayor autoridad y poder hacia la pareja, en jóvenes la conducta violenta se va dando de manera progresiva y muchas veces no se logra identificar las conductas como tal, ya que se idealiza a la pareja. Además, en esta construcción se define la violencia de pareja a toda conducta realizada por un miembro de la relación que va en contra de la integridad de la otra persona, se clasifican de tipo: violencia física, manipulación, coerción, violencia verbal y prohibición.

El objetivo principal de esta investigación fue la construcción y validación del "Cuestionario de Violencia de Pareja en Jóvenes", tener un instrumento con características psicométricas conocidas podría ser utilizado para identificar conductas violentas que se presentan durante la relación de pareja y reconocer los tipos de violencia existentes.

El análisis factorial que se realizó a partir de las respuestas de los participantes sugirió 5 factores que explicaban $53.5 \%$ de la varianza. El primer factor "Violencia Física" explicó el $12.62 \%$ de la varianza y agrupó a 10 ítems. El segundo factor: "Manipulación" explicó el $11.53 \%$ de la varianza y agrupó a 5 ítems. El tercer factor "Violencia Verbal" explicó el $10.94 \%$ de la varianza y agrupó a 6 ítems. El cuarto factor "Coerción" explicó el $9.25 \%$ de la varianza y agrupó a 3 ítems. El quinto factor "Prohibición" explicó el 9.16\% de la varianza y agrupó a 5 ítems. Finalmente, el instrumento quedó constituido por 29 ítems

En los resultados, se obtuvo un coeficiente de 0.937 en la medida de adecuación de muestreo (KMO) y la prueba de esfericidad de Bartlett de $<0.001$, lo que significa que es adecuado realizar el análisis factorial exploratorio.

Del mismo modo, con respecto a la fiabilidad, se alcanzó un puntaje de Alpha de Cronbach de .943 y Omega de McDonald's .947 en la escala global, lo que indica elevada fiabilidad. Así también, en los ítems se evidencia coeficientes de Cronbach que varían entre .940 a .942 los cuales afirman elevada fiabilidad y en el McDonald's .944 a .946 , el valor adecuado de la confiabilidad es de .70 y .90 (Campo y Oviedo, 2008). Además, el instrumento evidencia una buena consistencia interna y validez de constructo. 
En cuanto al instrumento psicométrico "Cuestionario de violencia de parejas en jóvenes" destacamos su facilidad de aplicación, pues es fácil de usar y de interpretar. Este instrumento puede constituirse en una herramienta para utilizarse con los jóvenes de manera colectiva, que permita la detección de la violencia hacia la pareja.

Por otra parte, para futuras investigaciones se recomienda ampliar los ítems en el cuarto factor. Asimismo, implementación de programas nacionales, locales en cuanto a la prevención y promoción en relación al respeto y la igualdad en las parejas jóvenes, lo cual contribuiría en las relaciones afectivas saludables.

\section{Declaración de financiamiento y de conflictos de interés:}

El estudio fue financiado por los autores, quienes declaran no tener algún tipo de conflicto de interés en la investigación realizada.

\section{Correspondencia}

Cristian Adriano Rengifo

Correo electrónico:

cristianadriano@upeu.edu.pe

\section{REFERENCIAS BIBLIOGRÁFICAS}

Bejarano, C. y Vega, L. (2014). Propiedades psicométricas de la Escala de Violencia en la Relación de Pareja en Jóvenes(E.V.R.P.-J.). (Tesis para licenciatura, Universidad Peruana Unión) Recuperado de: https://repositorio.upeu.edu. pe/bitstream/handle/UPEU/587/Cesia_Tesis_ bachiller_2015.pdf?sequence=1\&isAllowed=y.

Campo, A. y Oviedo, H. C. (2008). Propiedades psicométricas de una escala: la consistencia interna. Revista Salud Pública, 10 (5), 831839. Recuperado de https://www.redalyc.org/ pdf/422/42210515.pdf.

Escurra, M. y Salas, E. (2014). Construcción y validación del cuestionario de adicción a redes sociales (ARS). Liberabit, 20, (1), 73-91. Recuperado de: http://www.scielo.org.pe/pdf/ liber/v20n1/a07v20n1.pdf

García, M., Rodríguez, J. y Porcel, A. (2018). Diseño y validación de la escala para la detección de violencia en el noviazgo en jóvenes en la Universidad de Sevilla. Gaceta Sanitaria, 32(2), 121-128. Recuperado de: https:// www.sciencedirect.com/science/article/pii/ S0213911117302625?via\%3Dihub

Hernández, R., Fernández, C. y Baptista, M. (2010). Metodología de la Investigación, D.F., México. Editorial McGraw-Hill. Recuperado de: https://www.esup.edu.pe/descargas/ dep_investigacion/Metodologia $\% 20$ de $\% 20$ Ia $\% \overline{2}$ Oinvestigaci $\%$ C 3 \% B 3n \% 205 ta \% 20 Edici\%C3\%B3n.pdf

Instituto Nacional de Estadística e Informática. (2017). Perú: Indicadores de violencia familiar y sexual, 2000 - 2017. Recuperado de: https://www.inei. gob.pe/media/MenuRecursivo/publicaciones_ digitales/Est/Lib1465/libro.pdf .
León, M. (2017). Propiedades psicométricas del cuestionario de violencia entre novios en universitarios de Nuevo Chimbote-2017, (Tesis en Licenciatura, Universidad César Vallejo) . Recuperado de: http://repositorio. ucv.edu.pe/bitstream/handle/UCV/12510/leon_ $\mathrm{cm}$.pdf?sequence=1\&isAllowed=y

Lloret, S., Ferreres, A., Hernández, A. y Tomás, I. (2014). El análisis factorial exploratorio de los ítems: una guía práctica, revisada y actualizada, Anales de psicología. 30(3), 1151-1169. Recuperado de: http://scielo.isciii.es/pdf/ap/ v30n3/metodologia1.pdf.

Muñoz, J. y Echeburúa, E. (2016). Diferentes modalidades de violencia en la relación de pareja: implicaciones para la evaluación psicológica forense en el contexto legal español. Anuario de Psicología Jurídica, 26, 2-12. Recuperado de: https://www.sciencedirect.com/science/article/pii/ S1133074015000355

Pazos, M., Oliva, A. y Hernando, A. (2014). Violencia en relaciones de pareja de jóvenes y adolescentes. Revista latinoamericana de psicología, 46(3), 148-159. Recuperado de: https://www.sciencedirect.com/science/article/pii/ S0120053414700184

Rodríguez, J. (2007). Violencia hacia la pareja: revisión teórica. Psicopatología clínica, legal y forense, 7(1), 77-95. Recuperado de: https://dialnet. unirioja.es/servlet/articulo?codigo $=2553067$.

Rodríguez-Franco, L., López, J., Rodríguez, F., Bringas, C., Bellerín, A. y Estrada, C. (2010). Validación del Cuestionario de Violencia entre Novios (CUVINO) en jóvenes hispanohablantes: Análisis de resultados en España, México y Argentina. Revista Anuario de Psicología Clínica 
y de la Salud. 6 (2010), 45 - 52. Recuperado de:https://idus.us.es/xmlui/bitstream/ handle/11441/13939/Validaci\%c3\%b3n\%20 CUVINO.pdf?sequence $=2 \&$ isAllowed $=y$

Rubio, F., Carrasco, M., Amor, P. y Lopez-Gonzalez, M. (2015). Factores asociados a la violencia en el noviazgo entre adolescentes: una revisión crítica. Anuario de Psicología Jurídica, 25, 47-56. Recuperado de: https://www.sciencedirect.com/ science/article/pii/S1133074015000082

Sánchez, C. (2017). Conflictividad interpersonal en relaciones afectivas de noviazgo en adolescentes, (Tesis de Maestría Universidad de Oviedo) . Recuperado de: http://digibuo.
uniovi.es/dspace/bitstream/10651/45586/6/TFM CarlaSanchezTogneri.pdf.

Villena, E. (2016). Propriedades Psicométricas del Inventario de Violencia en las Relaciones de Noviazgo entre Adolescentes Estudiantes de la Provincia de Pacasmayo. Tesis de licenciatura, Universidad de César Vallejo) Recuperado de: http://repositorio.ucv.edu. pe/bitstream/handle/UCV/245/villena_ ve.pdf?sequence=1\&isAllowed=y.

Recibido: $17 / 02 / 2019$

Aceptado: $24 / 05 / 2020$ 